\title{
The Utility of TEM and AFM Techniques in the Preclinical Assessment of Nanomaterials
}

Jiwen Zheng, David Parmiter, Lesley Graham, Kunio Nagashima, Anil K. Patri and Scott E. McNeil

Nanotechnology Characterization Lab (NCL), SAIC-Frederick, Inc., NCI-Frederick, Frederick, Maryland 21702

Nanomaterials are showing great promise in their utility for biomedical applications, in areas such as drug delivery, diagnostics and image contrast enhancement. Nanoparticlebased formulations have demonstrated enhanced efficacy and decreased toxicity in comparison to conventional drugs. Comprehensive preclinical characterization of is a critical step in commercialization of nanoparticle-based formulations ${ }^{[1-5]}$. Nanoparticle formulations are often comprised of various compositions, including metals, metal oxides, polymers, lipids, biomolecules such as antibodies, proteins and DNA, and other organic compounds. In many cases, the nanoparticle combines two or more of these substances forming a conjugated, encapsulated or core/shell structure. The resulting complexity of nanoparticle-based formulations poses myriad characterization challenges.

Microscopy techniques such as transmission electron microscopy (TEM) and atomic force microscopy (AFM) have proved useful in the preclinical characterization of nanoparticle formulations, because they offer sub-nanometer scale resolution. When coupled with energy dispersive X-ray spectroscopy, TEM allows both observation of nanostructures and examination of their chemical composition. As a companion technique, AFM offers advantages for characterizing nanoparticles under physiological conditions, particularly for assessing surface characteristics including composition, conformation and activity.

This presentation covers the use of TEM and AFM to characterize a wide variety of nanomaterials both in their native state and in biological matrix (cells and tissues).

Funded by NCI Contract HHSN261200800001E.

References

1. S. E. McNeil, Journal of Leukocyte Biology, 78 (2005) 585.

2. J. B. Hall et al. Nanomedicine, 2 (2007) 789.

3. S. T. Stern et al. Toxicological Sciences, 106 (2008) 140.

4. M. A. Dobrovolskaia et al. Nanomedicine: Nanotechnology, Medicine, and Biology, 5 (2009) 106.

5. N. Sadrieh et al. Toxicological Sciences, in press. 